Check for updates

St George's, University of London, London, UK

Correspondence to: D Gill dgill@sgul.ac.uk

Cite this as: BMJ 2021;373:n1093 http://dx.doi.org/10.1136/bmj.n1093 Published: 29 April 2021

\section{We need clinical guidelines fit for a pandemic}

\author{
Guidance must be faster, bolder, and problem based \\ Dipender Gill, Emma H Baker, Andrew W Hitchings
}

The covid-19 pandemic unleashed itself onto healthcare services at pace. Evidence evolved, ${ }^{12}$ opinions conflicted, ${ }^{3}$ and both real and perceived resource constraints were confronted. ${ }^{4}$ Against this backdrop, clinicians had to make time-critical decisions about what treatments to offer, or not, to their patients.

Working groups were hastily convened, but much of the decision making happened at the bedside. Undoubtedly, many tackled the same questions simultaneously but reached different answers. Centrally produced guidance was conspicuously slow to emerge. For example, by the time the National Institute for Health and Care Excellence (NICE) published a guideline on the management of covid-19 on 23 March 2021, 5 UK clinicians had managed more than 450 ooo hospital admissions related to the disease. $^{6}$

\section{Uncertainty paradox}

The imperative for guidance is greatest when evidence is limited or evolving. Yet this is when guidance producers are often most reluctant to be bold, defaulting to a negative recommendation or none at all. The view that this is a precautionary approach is illusory. A decision not to make a firm recommendation centrally is really just a decision to defer it locally. Given the variation in clinicians' expertise, time, and resources to formulate their own protocols, heterogeneity in care and inequalities in access to treatment inevitably follow.

Many of the challenges presented to clinicians and policy makers have required high stakes, costly, or ethically sensitive judgments. For example, in response to realistic concern that demand for intensive care facilities could outstrip supply, much work was done to develop triage processes that would be fair, transparent, and ethically justified. ${ }^{7}$ However, the output from this work varied substantially in content and quality. ${ }^{7}$

Other judgments were lower in profile but still demanding of clinicians' time and cognitive bandwidth. For example, the prothrombotic phenotype of the disease, particularly in critical illness, led many hospitals and professional bodies ${ }^{8}$ to develop bespoke protocols for thromboprophylaxis in covid-19. National guidance on this (published in November $2020^{9}$ ) was too slow to avert substantial variation in practice.

Use of tocilizumab and sarilumab in covid-19 was similarly variable. NICE published guidance on this in April 2021, ${ }^{5}$ around two months (and 30000 UK hospital admissions ${ }^{6}$ ) after results were made public from a pivotal trial. ${ }^{10}$ Unanswered questions still remain that present dilemmas in practice-for example, on the value of a second dose, an element of the trial protocol that was deferred to the judgment of treating clinicians. ${ }^{10}$

\section{More agility}

Although controversial, ${ }^{11}$ the UK government's decision to delay the second dose of covid-19 vaccines has shown that policy makers and their scientific advisers are willing to apply expert judgment to time sensitive decisions. Likewise, the Medicines and Healthcare Products Regulatory Agency's decision to authorise the Oxford-AstraZeneca vaccine for people older than 65 years $^{12}$ despite limited clinical data for this population at the time.

Guidance producers must act similarly, leveraging clinical and scientific opinion to produce clear guidance that is informed by evidence but not constrained by its absence. The traditional model of comprehensive topic guidelines should also be reconsidered. These take years to develop and generally do not meet the needs of users, who are more likely to "dip in" for answers to specific questions than read a guideline exhaustively. Disaggregating guidance by question rather than topic will accelerate production and updates. The signal from NICE, in its recently launched five year strategy, ${ }^{13}$ that it will move towards dynamic, modular guidelines is encouraging. Likewise, its adoption of a "living guideline" platform for covid-19.

Guidance is worthwhile only if it is incorporated into practice, and guidelines are often criticised for prioritising standardisation over the needs of individual patients. ${ }^{14}$ However, clinicians must be mindful that the decisions they make for their patients may impose costs for others. These costs may be direct, and occasionally identifiable, if they relate to the allocation of the same constrained resource (such as a drug). More often they are indirect, resulting from unidentified opportunity costs and the unappreciated downstream consequences of variation in practice. Well produced national guidance brings inherent benefits in reducing unwarranted variation, as well as accounting for system factors such as cost and supply, which may be impossible to take into consideration at the frontline.

The pandemic has brought the limitations of the traditional clinical guidance model into sharp focus and highlighted opportunities to improve it. We welcome the promise from NICE of a move towards more rapid, modular, and living recommendations. We urge guidance producers also to confront the paradox that the imperative for guidance is greatest when evidence is scarce. Finally, clinicians and decision makers at the frontline need to reciprocate this effort by implementing national 
recommendations. These are not an affront to our professional judgment but a positive response to the collective clinical uncertainty.

Competing interests: We have read and understood BMJ policy on declaration of interests and declare the following interests: DG is employed part time by Novo Nordisk and has received consultancy fees from Policy Wisdom, unrelated to the submitted work. AWH is a member of a technology appraisal committee and a clinical guideline committee for NICE and chairs a local formulary committee.

Provenance and peer review: Not commissioned; externally peer reviewed.

We thank Stephen Burgess for his comments on an early draft.

1 Rochwerg B, Siemieniuk RA, Agoritsas T, etal. A living WHO guideline on drugs for covid-19. BMJ 2020;370:m3379. doi: 10.1136/bmj.m3379. pmid: 32887691

2 Siemieniuk RA, Bartoszko JJ, Ge L, etal. Drug treatments for covid-19: living systematic review and network meta-analysis. BMJ2020;370:m2980. doi: 10.1136/bmj.m2980. pmid: 32732190

3 Nagler RH, Vogel RI, Gollust SE, Rothman AJ, Fowler EF, Yzer MC. Public perceptions of conflicting information surrounding COVID-19: Results from a nationally representative survey of US adults. PLoS One 2020;15:e0240776. doi: 10.1371/journal.pone.0240776. pmid: 33085719

4 Mahase E. Covid-19: Hospitals in crisis as ambulances queue and staff are asked to cancel leave. BMJ2020;371:m4980. doi: 10.1136/bmj.m4980. pmid: 33384317

5 NICE. covid-19 rapid guideline: managing covid-19. 2021 https://www. nice.org.uk/guidance/ng191

6 Public Heath England. Coronavirus (COVID-19) in the UK. 2021. https://coronavirus.data.gov.uk/details/healthcare

7 Tyrrell CSB, Mytton OT, Gentry SV, etal. Managing intensive care admissions when there are not enough beds during the COVID-19 pandemic: a systematic review. Thorax 2021;76:302-12. doi: 10.1136/thoraxjnl-2020-215518. pmid: 33334908

8 Royal College of Physicians. What to consider about heparin anticoagulation in patients admitted to hospital with covid-19. 2021 https://www.rcplondon.ac.uk/file/28126/download

9 NICE. Covid-19 rapid guideline: reducing the risk of venous thromboembolism in over $16 \mathrm{~s}$ with covid-19. 2020. https://www.nice.org.uk/guidance/ng186

10 Horby PW, Pessoa-Amorim G, Peto L, etal. Tocilizumab in patients admitted to hospital with COVID-19 (RECOVERY): preliminary results of a randomised, controlled, open-label, platform trial.medRxiv 2021:2021.02.11.21249258. doi: 10.1101/2021.02.11.21249258

11 Mahase E. Covid-19: Order to reschedule and delay second vaccine dose is "totally unfair," says BMA. BMJ 2020;371:m4978. doi: 10.1136/bmj.m4978. pmid: 33384299

12 Medicines and Healthcare Products Regulatory Agency. Information for healthcare professionals on covid-19 vaccine AstraZeneca. 2021. https://www.gov.uk/government/publications/regulatoryapproval-of-covid-19-vaccine-astrazeneca/information-for-healthcare-professionals-on-covid19-vaccine-astrazeneca

13 NICE. The NICE strategy 2021 to 2026. 2021. https://www. nice.org.uk/about/who-we-are/corporate-publications/the-nice-strategy-2021-to-2026

14 Bion JF, Abrusci T, Hibbert P. Human factors in the management of the critically ill patient. Br J Anaesth 2010;105:26-33. doi: 10.1093/bja/aeq126 pmid: 20511333

This article is made freely available for use in accordance with BMJ's website terms and conditions for the duration of the covid-19 pandemic or until otherwise determined by BMJ. You may use, download and print the article for any lawful, non-commercial purpose (including text and data mining) provided that all copyright notices and trade marks are retained. 